\title{
Obtención de mapas de humedad del combustible a partir de variables meteorológicas para la predicción del riesgo de incendios forestales a escala regional: nuevo enfoque a los actuales índices de peligro de incendio
}

Obtaining fuel moisture maps from meteorological variables for the prediction of fire risk on a regional scale: a new approach to the current fire danger indexes

Cunill Camprubí, A. ${ }^{*}$

'1Escola Tècnica Superior d'Enginyeria Agrària Universitat de Lleida. Avinguda Rovira Roure, 191. 25198. Lleida 


\title{
Resumen
}

Los incendios forestales constituyen una de las mayores perturbaciones forestales a nivel mundial y, en especial, en las regiones mediterráneas. Este trabajo propone la aplicación espacial de un método de predicción del contenido de humedad del combustible fino y muerto (FM), que muestra una relación contrastada con el área quemada acumulada tras un incendio. El modelo empleado para predecir FM requiere únicamente del déficit de presión de vapor, cuyo valor se obtiene de los datos en cuadrícula de temperatura y humedad relativa. Para convertir las mediciones puntuales adquiridas de la red de estaciones meteorológicas a superficies continuas, se probaron las siguientes técnicas de interpolación espacial: regresión lineal (RL), ponderación por el inverso de la distancia (IDW) y kriging ordinario (OK). Dentro de la RL se evaluaron dos funciones diferentes con la elevación, la latitud y la longitud como variables de entrada. El estudio se realizó en el territorio español de la Península Ibérica y las Islas Baleares durante junio y julio de 2018. Los mejores resultados se obtuvieron con la técnica de OK. El producto final es un aplicativo automatizado para la estimación del contenido de humedad de los combustibles finos muertos, que busca ser operativo entre los cuerpos de extinción de incendios forestales como alerta anticipada a la ocurrencia de grandes incendios.

Palabras clave: déficit de presión de vapor, geoestadística, humedad del combustible, interpolación espacial.

\begin{abstract}
Forest fires constitute one of the major forest disturbances worldwide and, especially, in Mediterranean regions. This work proposes the spatial application of a method to estimate dead fine fuel moisture content (FM), that shows a contrasted relationship with the cumulative burned area after a fire. The model used to predict FM requires only the vapor pressure deficit, whose value is obtained from the gridded data of temperature and relative humidity. To convert the point data acquired from the Spanish meteorological station network to continuous surfaces, the following spatial interpolation techniques were tested: linear regression (RL), inverse distance weighted (IDW) and ordinary kriging (OK). Within the RL, two different functions were evaluated with elevation, latitude and longitude as input variables. The study was performed in the Spanish Iberian Peninsula and the Balearic Islands, and during June and July 2018. The best results were obtained with the OK method. The final product is an automated application for daily estimates of the moisture content of dead fine fuels that can be readily used for operational purposes as part of an early warning system of the potential for large fires.
\end{abstract}

Keywords: fuel moisture, geostatistics, spatial interpolation, vapor pressure deficit. 


\section{Introducción}

Los incendios forestales son un fenómeno recurrente de los paisajes forestales a nivel global (Boer et al., 2017). En las regiones mediterráneas, su régimen tradicional se ha visto alterado por causas como el incremento de la superficie forestal y el cambio climático (Pausas \& Fernández-Muñoz, 2012). Por ello, es necesario contar con un sistema capaz de predecir, con cierta exactitud y precisión, dónde y cuándo se puede desarrollar un incendio, a fin de poder anticiparse estratégicamente al problema (Chuvieso y Martín, 2004).

Se ha demostrado que los índices de sequía empleados actualmente para estimar el peligro de incendios, como el Canadian Forest Fire Weather Index (FWI), tienen capacidades limitadas para pronosticar la disponibilidad de los combustibles (Castellnou et al., 2005; Martin Soler et al., 2017). Su uso se ha extendido a regiones cuyas condiciones ambientales difieren mucho de las cuales se desarrolló el sistema (Aguado et al., 2007; Chuvieso, 2003). También, se ha visto un aumento en la frecuencia de grandes incendios fuera de estación (Chuvieso y Martín, 2004), como el ocurrido en Pedrogão Grande (Portugal) en 2017, donde la situación crítica tuvo lugar a principios de junio y el índice FWI no proporcionó una advertencia temprana de la situación (Boer et al., 2017).

Resco de Dios et al. (2015) desarrollaron un modelo semi-mecanicista que predice la humedad de los combustibles finos muertos (FM; $<25.4 \mathrm{~mm}$ ) basado en el déficit de presión de vapor (D) atmosférico, que proporciona resultados precisos en un amplio rango de lugares y ecosistemas. FM responde a D por efecto de la difusión del vapor de agua (Nelson, 2001) y está estrechamente vinculada con la probabilidad de ignición y propagación inicial de un fuego (Rothermel, 1983). De hecho, se ha cuantificado una relación entre FM y el área quemada en diferentes ecosistemas (Nolan et al., 2016a), incluidos los de la Península Ibérica (Boer et al., 2017), que ha permitido determinar los niveles críticos de humedad a partir de los cuales ocurren grandes incendios forestales.

El objetivo principal de este trabajo fue desarrollar un método automatizado para estimar FM diariamente a escala regional mediante el uso del modelo de Resco de Dios et al. (2015) y según los parámetros propuestos por Nolan et al. (2016b). Para ello, el objetivo específico consistió en evaluar las tres técnicas de interpolación más utilizadas en la literatura (Li \& Heap, 2011); la regresión lineal (RL), el método de ponderación por el inverso de la distancia (IDW) y el método geoestadístico de kriging ordinario (OK). El rendimiento de estos métodos depende de las propiedades que deben cumplirse para cada uno de ellos (Li \& Heap, 2014). Por ello, se llevó a cabo un análisis exploratorio previo y un análisis espacial para establecer si los datos cumplían con los requisitos. Los mapas generados con la mejor técnica pretenden determinar, a tiempo casi-real y con mayor exactitud, el riesgo de grandes incendios en cada punto de la Península Ibérica y Baleares en función de los umbrales críticos nombrados anteriormente y definidos, en este caso, por Boer et al. (2017). 


\section{Material y métodos}

\section{1. Área de estudio}

El área de estudio se centró en la España peninsular y las Islas Baleares, que en conjunto cubren una superficie de $502494 \mathrm{~km}^{2}$. Este territorio se divide en dos biomas (Olson et al., 2001): el bioma de los bosques templados de hoja ancha y mixtos, y el bioma de los bosques y matorrales mediterráneos. Localmente, también existen zonas con climas fríos restringidas en áreas de montaña.

\subsection{Predicción de la humedad del combustible}

Para estimar FM, se empleó el modelo de Resco de Dios et al. (2015) calibrado por Nolan et al. (2016b) (Ec.1):

$$
F M_{D}(\%)=6.79+27.43 e^{(-1.05 D)}
$$

D se obtuvo del cálculo de la presión de saturación del vapor de agua y la presión real de vapor de agua, en función de la temperatura máxima diaria del aire (Tmáx) y la humedad relativa correspondiente al momento de Tmáx. Siguiendo a Cáceres et al. (2018), esta última se convirtió a temperatura en el punto de rocío (Tpr) para mejorar los resultados. El análisis y los ajustes de los métodos se hicieron con el registro diario de datos meteorológicos en el periodo entre el 1 de junio y el 31 de julio de 2018, obtenidos del servicio AEMET OpenData de la Agencia Estatal de Meteorología (AEMET, 2018). El Modelo Digital de Elevaciones (MDE) de España (IGN, 2018) se empleó como límite y base para las interpolaciones.

\subsection{Análisis exploratorio}

El análisis estadístico exploratorio consistió en describir los datos por medio de técnicas clásicas. También, se evaluó la representatividad del área de estudio en cuanto a la distribución espacial de los puntos de muestreo (red de estaciones) y sus elevaciones mediante el Análisis de Patrones de Puntos (Cabrero Ortega y García Pérez, 2015) y la prueba de contraste de Kolmogorov-Smirnov en comparación con las elevaciones del MDE.

\subsection{Análisis espacial}

El análisis espacial se hizo a través del variograma muestral. Este gráfico representa la disimilitud o semivarianza de una variable en función de la distancia entre puntos de muestreo (Cressie, 1993). Se usó para determinar el grado de autocorrelación espacial y comprobar los supuestos de estacionariedad y anisotropía de los métodos basados en distancias. Esto es, que la variable de interés provenga de un 
proceso aleatorio continuo en el espacio, cuya varianza entre dos puntos no dependa de su localización ni de la dirección que se tome (Olaya, 2014).

Seguidamente, se ajustó una función matemática (Webster \& Oliver, 2007) que permite conocer las semivarianzas para cualquier distancia y sirve como parte integrante de los métodos basados en la geoestadística. En este caso, también se usó para descartar valores topológicamente discordantes siguiendo el algoritmo z descrito por Lu et al. (2003). Una vez descartados se repitió el proceso de ajuste.

\subsection{Obtención de los mapas}

Los métodos de interpolación se aplicaron a Tmáx y Tpr antes del cálculo de FM. Como métodos clásicos, se probaron dos modelos de regresión lineal múltiple (RL1; Ec.2 y RL2; Ec.3) basados en la longitud (x), la latitud (y) y la elevación (e):

$$
\begin{gathered}
Z=\beta_{0}+\beta_{1} e+\beta_{2} x+\beta_{3} y+\varepsilon \\
Z=\beta_{0}+\beta_{1}\left(\frac{e}{10^{3}}\right)+\beta_{2}\left(\frac{x}{10^{5}}\right)+\beta_{3}\left(\frac{y}{10^{5}}\right)+\beta_{4}\left(\frac{e}{10^{3}}\right)^{2}+\beta_{5}\left(\frac{x}{10^{5}}\right)^{2}+\beta_{6}\left(\frac{y}{10^{5}}\right)^{2}+\varepsilon
\end{gathered}
$$

donde $Z$ es la variable de interés, $\beta_{0}$ y $\beta_{\mathrm{n}>0}$ son la intercepción y los coeficientes de la regresión, respectivamente, y $\mathcal{E}$ es el error aleatorio de la predicción. El segundo fue desarrollado por Sánchez-Palomares et al. (1999) para estimar la temperatura en la Península Ibérica. Los métodos basados en distancias, IDW y OK, se aplicaron siguiendo las indicaciones de Bivand et al. (2003) y Hengl (2009). Para evaluar las predicciones de estos últimos se hizo una validación cruzada del tipo leave-one-out cross-validation (LOO).

Para compararlos, se cuantificó el rendimiento a través del cálculo del error medio (MBE), el error absoluto medio (MAE), la raíz del error cuadrático medio (RMSE) y el coeficiente de determinación $\mathrm{R}^{2}$. Adicionalmente, se estimó el error de FM derivado de MAE de ambas variables según el valor promedio del percentil P50 y P95 de sus distribuciones.

Los análisis y la obtención automática de los mapas se realizó con los paquetes integrados del lenguaje de programación estadística R (R Core Team, 2018) y las librerías externas meteoland (Cáceres et al., 2018), plantecophys (Duursma, 2015), spatstat (Baddeley \& Turner, 2005), gstat (Pebesma, 2004) y rgdal (Bivand et al., 2018).

\section{Resultados y discusión}

\subsection{Análisis exploratorio}

En la exploración previa de los datos se detectó, en mayor o menor grado, la existencia de poblaciones distintas en sus distribuciones, que podrían coincidir con 
las regiones bioclimáticas que ocupan el territorio. Esta circunstancia viola parte de la hipótesis de estacionariedad (Cressie, 1993).

Por otro lado, el número promedio de puntos de muestreo fue de 706 y 703 para Tmáx y Tpr, respectivamente. Se cumplía con el mínimo aconsejado por Webster \& Oliver (2007) de 150 puntos para una estimación aceptable del variograma muestral. El análisis de patrones de puntos sugirió un patrón de distribución homogéneamente distribuido al azar por toda el área de estudio. La representatividad de elevaciones fue estadísticamente similar a la del $\operatorname{MDE}(\mathrm{P}=0.06>0.05)$, con un valor de correlación de Pearson de 0.84 . Por todo esto, se consideró una representatividad adecuada para capturar los cambios en las distintas escalas de variación.

\subsection{Análisis espacial}

Los variogramas muestrales de la Tmáx mostraron un incremento inicial lineal, constante y monotónico de la semivarianza hasta alcanzar aproximadamente una distancia de $200 \mathrm{~km}$. Esto confirma la existencia de autocorrelación espacial. A esa distancia se producía un aumento generalizado más o menos brusco de la semivarianza sin llegar a un máximo constante o asintótico. Según Webster \& Oliver (2007), esto es signo de tendencias a gran escala superpuestas a la variación aleatoria a escala local, lo que concuerda con las estructuras diferenciales observadas en el análisis exploratorio. En el caso de la Tpr, el comportamiento fue similar a la Tmáx. La aparente estabilización de la varianza se alcanzaba entre los $200 \mathrm{~km}$ y los $350 \mathrm{~km}$, aproximadamente.

Del análisis anisotrópico se obtuvo que los variogramas direccionales presentaban distintos grados de varianza entre ellos atribuible al mismo hecho (Webster $\&$ Oliver, 2007). Estas circunstancias llevaron a adoptar un enfoque localizado a $250 \mathrm{~km}$, donde se asumía estacionariedad a nivel local y la anisotropía no parecía tan evidente.

La proporción de valores atípicos espaciales extraídos giró en torno al $1.2 \%$ y al $1.3 \%$ para la Tmáx.y la Tpr, respectivamente. Su extracción supuso una reducción media del error de estimación del 5.8\% y del 7.1\%, obtenido tras una validación cruzada hecha con el método de $\mathrm{OK}$.

\subsection{Interpolación}

Los valores promedio de los resultados obtenidos en la evaluación del rendimiento de los distintos métodos se recogen en la Tabla 1.

La técnica de OK mostró una precisión superior en ambas variables. En general, los valores de MAE no se alejaron mucho de los valores de RMSE y MBE fue cercano a 0 en todos los métodos, lo cual apunta a una escasa presencia de errores relativamente altos en las estimaciones y que estas no presentaron un sesgo significativo.

En conjunto, la capacidad explicativa de los distintos métodos fue menor para la Tpr. Nuevamente, OK mostró mejor rendimiento que los demás. En la Tmáx, se 
Tabla 1. Error absoluto medio (MAE), raíz del error cuadrático medio (RMSE), error medio (MBE) y coeficiente de determinación $\left(R^{2}\right)$ de los distintos métodos de interpolación. Valores promedio para la temperatura máxima (Tmáx) y la temperatura en el punto de rocío (Tpr) en el periodo de estudio.

\begin{tabular}{ccccccccc}
\hline \multirow{2}{*}{ Método } & Tmáx $\left({ }^{\circ} \mathbf{C}\right)$ & \multicolumn{7}{c}{ Tpr $\left({ }^{\circ} \mathbf{C}\right)$} \\
\cline { 2 - 9 } & MAE & RMSE & MBE & $\boldsymbol{R}^{2}$ & MAE & RMSE & MBE & $\boldsymbol{R}^{2}$ \\
\hline RL1 & 2.347 & 2.931 & $-1.5 \mathrm{e}-17$ & 0.476 & 2.313 & 3.027 & $6.9 \mathrm{e}-18$ & 0.450 \\
\hline RL2 & 1.734 & 2.208 & $1.4 \mathrm{e}-17$ & 0.707 & 2.111 & 2.793 & $-7.8 \mathrm{e}-18$ & 0.529 \\
\hline IDW & 1.476 & 1.905 & 0.061 & 0.767 & 1.757 & 2.342 & -0.036 & 0.630 \\
\hline OK & 1.402 & 1.819 & -0.003 & 0.786 & 1.674 & 2.224 & -0.010 & 0.663 \\
\hline
\end{tabular}

hizo evidente el mal funcionamiento de RL1. El análisis de los residuos desveló que incumplía los supuestos de linealidad y normalidad de la regresión lineal. Con OK, el error relativo estimado en FM fue del 5.4\% y del 2.3\% para P50 y P95 de Tmáx, respectivamente, y del $4.8 \%$ y $12.4 \%$ para Tpr. Estos resultados coinciden con los obtenidos por Resco de Dios et al. (2015), que demuestran que el modelo de predicción funciona mejor en el rango de FM más crítico para la ocurrencia de grandes incendios.

La Figura 1 ilustra un ejemplo donde se observan los cambios de humedad que registra el modelo FMD producidos a gran escala en un lapso relativamente corto, acordes con Nolan et al. (2016a).
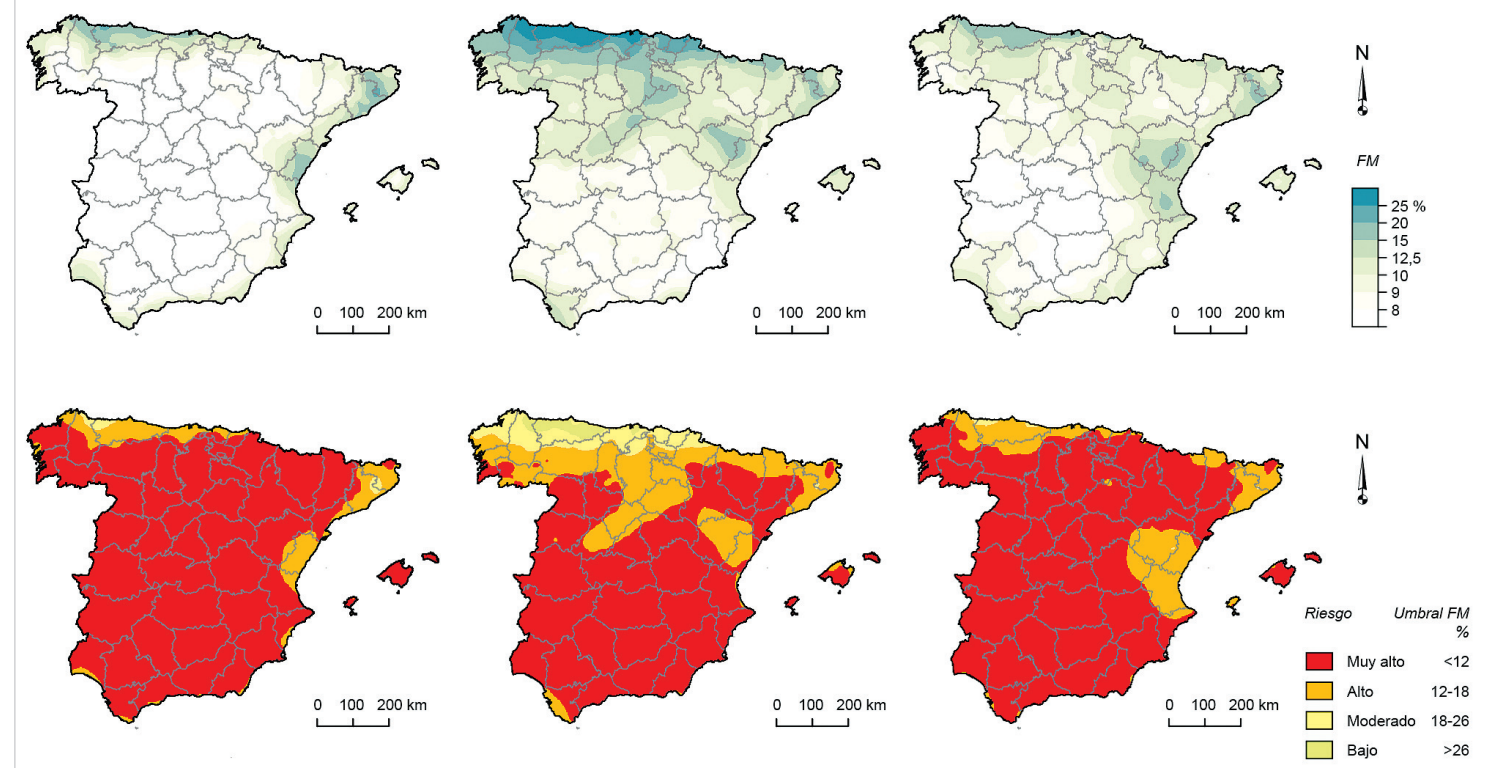

3 de septiembre

6 de septiembre

8 de septiembre

Figura 1. Mapas de contenido de humedad de los combustibles finos muertos (FM) generados con kriging ordinario y niveles de riesgo de incendio asociados a los umbrales críticos definidos por Boer et al. (2017). 


\section{Conclusiones}

En este proyecto se ha desarrollado un método que proporciona mapas del contenido de humedad de los combustibles finos muertos de forma regionalizada, poniendo en práctica un modelo semi-mecanicista simple basado en la demanda evaporativa de la atmósfera y que requiere, únicamente, de registros de temperatura y humedad relativa.

Con estos mapas se puede determinar el riesgo meteorológico de ocurrencia de incendio para todo el territorio a través de unos umbrales que relacionan FM con el incremento de área quemada tras un incendio.

De las tres técnicas de interpolación empleadas para generar las superficies continuas a partir de los registros puntuales de las variables meteorológicas, la técnica OK mostró ser la más precisa y efectiva, con un error estimado en FM comparable al error que se puede cometer en su medición real.

Con esto, se busca tener una herramienta que, de forma automatizada, actualice el valor de FM diariamente en todo el territorio español y sirva como alerta temprana frente a situaciones de probabilidad extrema de gran incendio forestal.

\section{Agradecimientos}

A Víctor Resco de Dios, por proponerme este reto y confiar en mí desde un principio.

\section{Bibliografía}

AEMET. 2018. AEMET Open Data. Sistema para la difusión y reutilización de la información de la Agencia Estatal de Meteorología. https://opendata.aemet.es/centro dedescargas/inicio

Aguado, I.; Chuvieco, E.; Borén, R.; Nieto, H.; 2007. Estimation of dead fuel moisture content from meteorological data in Mediterranean areas. Applications in fire danger assessment. Int. J. Wildland Fire, 16(4), 390-397. https://doi.org/10.1071/WF06136

Baddeley, A.; \& Turner, R.; 2005. spatstat: An R Package for Analyzing Spatial Point Patterns. J. Stat. Softw., 12(6), 1-42. https://doi.org/10.18637/jss.v012.i06

Bivand, R.; Keitt, T.; \& Rowlingson, B.; 2018. rgdal: Bindings for the 'Geospatial' Data Abstraction Library. R package version 1.3-3. https://CRAN.R-project.org/package=rgdal.

Bivand, R.; Pebesma, E.; \& Gómez-Rubio, V.; 2013. Applied Spatial Data Analysis with R. Second Edition. Springer, New York. https://doi.org/10.1007/978-1-4614-7618-4

Boer, M.M.; Nolan, R.H.; Resco De Dios, V.; Clarke, H.; Price, O.F.; \& Bradstock, R.A.; 2017. Changing Weather Extremes Call for Early Warning of Potential for Catastrophic Fire. Earth's Future, 5(12), 1196-1202. https://doi.org/10.1002/2017EF000657

Cabrero Ortega, Y.; García Pérez, A.; 2015. Análisis estadístico de datos espaciales con QGIS y R. Universidad Nacional de Educación a Distancia UNED, Madrid. 
Cáceres, M.; De Martin, N.; Granda, V.; \& Cabon, A.; 2018. Meteoland: Landscape Meteorology Tools. $\mathrm{R}$ package version 0.7.1.

Castellnou, M.; Miralles, M.; Molina, D.; 2005. Análisis de la disponibilidad de combustible: índices meteorológicos críticos para la ocurrencia de cada patrón de grandes incendios forestales en la zona de Tivissa. IV Congreso Forestal Español. Zaragoza, (septiembre 2017).

Chuvieso, E.; 2003. Wildland fire danger. Estimation and mapping. The role of remote sensing data. World Scient, River Edge, N.J. https://doi.org/10.1142/9789812791177

Chuvieso, E.; Martín, M. del P.; 2004. Nuevas tecnologías para la estimación del riesgo de incendios forestales. Consejo Superior de Investigaciones Científicas, Madrid.

Cressie, N.; 1993. Statistics for Spatial Data (Revised Ed). Wiley-Interscience Publication, USA. https://doi.org/10.1002/9781119115151

Duursma, R.A.; 2015. Plantecophys - An R Package for Analysing and Modelling Leaf Gas Exchange Data. PLoS ONE, 10(11), e0143346. https://doi.org/http://doi.org/10.1371/ journal.pone. 0143346

Hengl, T.; 2009. A Practical guide to Geostatistical Mapping of Environmental Variables. Sci. and Tech. Res. ser., EU.

IGN. 2018. Instituto Geográfico Nacional. Modelo Digital de Elevaciones de España. MD T200. http://centrodedescargas.cnig.es/Centro Descargas/catalogo.do?Serie=LIDAR\#

Li, J.; Heap, A.D.; 2011. A review of comparative studies of spatial interpolation methods in environmental sciences: Performance and impact factors. Ecol. Informatics, 6(3-4), 228-241. https://doi.org/10.1016/j.ecoinf.2010.12.003

Li, J.; Heap, A.D.; 2014. Spatial interpolation methods applied in the environmental sciences: A review. Environ. Model. and Softw., 53, 173-189. https://doi.org/10.1016/ j.envsoft.2013.12.008

Lu, C.-T.; Chen, D.; Kou, Y.; 2003. Algorithms for spatial outlier detection. Third IEEE International Conf. on Data Mining, 0-3. https://doi.org/10.1109/ICDM.2003.1250986

Martin Soler, M.; Bonet, J. A.; Martínez De Aragón, J.; Voltas, J.; Coll, L.; Resco De Dios, V.; 2017. Crown bulk density and fuelmoisture dynamics in Pinus pinaster stands are neither modified by thinning nor captured by the Forest FireWeather Index. Annals of For. Sci. https://doi.org/10.1007/s12192-010-0223-9

Nelson, R.M.; 2001. Water Relations of Forest Fuels. In: Johnson, E. \& Miyanishi, K (eds.), Forest Fires: Behavior and Ecological Effects. Elsevier, pp. 79-149. https://doi.org/ 10.1016/B978-012386660-8/50006-4

Nolan, R.H.; Boer, M.M.; Resco De Dios, V.; Caccamo, G.; Bradstock, R.A.; 2016a. Largescale, dynamic transformations in fuel moisture drive wildfire activity across southeastern Australia. Geophys. Res. Lett., 43(9), 4229-4238. https://doi.org/10.1002/201 6GL068614

Nolan, R.H.; Resco de Dios, V.; Boer, M.M.; Caccamo, G.; Goulden, M.L.; Bradstock, R.A.; 2016b. Predicting dead fine fuel moisture at regional scales using vapour pressure deficit from MODIS and gridded weather data. Remote Sens. of Environ., 174, 100 108. https://doi.org/10.1016/j.rse.2015.12.010

Olaya, V.; 2014. Sistemas de Información Geográfica. https://volaya.github.io/libro-sig/

Olson, D.M.; Dinerstein, E.; Wikramanayake, E.D.; Burgess, N.D.; Powell, G.V.N.; Underwood, E.C.; ... Kassem, K.R.; 2001. Terrestrial Ecoregions of the World: A New Map of Life on Earth. BioScience, 51(11), 933. https://doi.org/10.1641/0006-3568(2001) 
051[0933:TEOTWA]2.0.CO;2

Pausas, J.G.; Fernández-Muñoz, S.; 2012. Fire regime changes in the Western Mediterranean Basin: from fuel-limited to drought-driven fire regime. Clim. Change, 110(1-2), 215-226. https://doi.org/10.1007/s10584-011-0060-6

Pebesma, E.J.; 2004. Multivariable geostatistics in S: the gstat package. Comput. \& Geosci., 30: 683-691. https://doi.org/10.1016/j.cageo.2004.03.012

R Core Team. 2018. R: A language and environment for statistical computing. R Foundation for Statistical Computing, Vienna, Austria. URL https://www.R-project.org/.

Resco de Dios, V.; Fellows, A.W.; Nolan, R.H.; Boer, M.M.; Bradstock, R.A.; Domingo, F.; Goulden, M.L.; 2015. A semi-mechanistic model for predicting the moisture content of fine litter. Agric. and For Meteorol, 203, 64-73. https://doi.org/10.1016/j.agrformet. 2015.01.002

Rothermel, R.C.; 1983. How to predict the spread and intensity of forest and range fires. Gen. Tech. Rep. INT-143. Ogden, UT: U.S. https://doi.org/10.2737/INT-GTR-143

Sánchez-Palomares, O.; Sánchez-Serrano, F.; Carretero, M.P.; 1999. Modelos y Cartografía de estimaciones climáticas termopluviométricas para la España peninsular. Ministerio de Agricultura, Pesca y Alimentación. Instituto Nacional de Investigación y Tecnología Agraria y Alimentaria, Madrid.

Webster, R.; Oliver, M.A.; 2007. Geostatistics for Environmental Scientists. Statistics in Practise. Second ed. John Wiley \& Sons, Chichester. https://doi.org/10.1002/9780 470517277 